\title{
Genome Sequence Data of Peronophythora litchii, an Oomycete Pathogen Causing Litchi Downy Blight
}

\author{
Hengyuan Guo, ${ }^{1}$ Jiandong Bao, ${ }^{2}$ Lianyu Lin, ${ }^{2}$ Zhixin Wang, ${ }^{1}$ Mingyue Shi, ${ }^{3}$ Yuting Huang, ${ }^{3}$ \\ Rongbo Wang, ${ }^{3}$ Benjin Li, ${ }^{3}$ Peiqing Liu, ${ }^{3}$ and Qinghe Chen ${ }^{1,3, \dagger}$ \\ ${ }^{1}$ Key Laboratory of Green Prevention and Control of Tropical Plant Diseases and Pests, Ministry of \\ Education, College of Plant Protection, Hainan University, Haikou 570228, China \\ ${ }^{2}$ Fujian University Key Laboratory for Plant-Microbe Interaction, College of Life Sciences, Fujian \\ Agriculture and Forestry University, Fuzhou, 350002, China \\ ${ }^{3}$ Fujian Key Laboratory for Monitoring and Integrated Management of Crop Pests, Institute of Plant \\ Protection, Fujian Academy of Agricultural Sciences, Fuzhou 350003, China
}

\begin{abstract}
Peronophythora litchii is an oomycete pathogen that exclusively infects litchi, with infection stages affecting a broad range of tissues. In this study, we obtained a near chromosome-level genome assembly of $P$. litchii ZL2018 from China using Oxford Nanopore Technologies long-read sequencing and Illumina short-read sequencing. The genome assembly was $64.15 \mathrm{Mb}$ in size and consisted of 81 contigs with an $\mathrm{N}_{50}$ of $1.43 \mathrm{Mb}$ and a maximum length of $4.74 \mathrm{Mb}$. Excluding $34.67 \%$ of repeat sequences, 14,857 protein-coding genes were identified, among which 14,447 genes were annotated. We also predicted 306 candidate RxLR effectors in the assembly. The high-quality genome assembly and annotation resources reported in this study will provide new insight into the infection mechanisms of $P$. litchii.
\end{abstract}

Litchi (Litchi chinensis Sonn.) is a commercially important subtropical fruit; however, its production and quality are restricted by litchi downy blight, which is caused by the pathogen Peronophythora litchi (Chi et al. 1984; Vien et al. 2001). Peronophythora litchi is among the major diseases of litchi, with commercial losses of more than $60 \%$ reported in Southern China (Ann and Ko 1984; Huang et al. 1983; Wang et al. 2009a). Peronophythora litchii is an oomycete pathogen that exclusively infects litchi, with infection stages affecting a broad range of tissues, including leaves, panicles, mature fruit, new shoots, and even extending to postharvest transportation and storage (Kao and Leu 1980; Vien et al. 2001).

Peronophythora litchii infects litchi leaves or fruits through oospores, which subsequently develop into sporangia that release zoospores into the litchi orchard. Oomycete infection initially causes withering and watery brown spots at infection sites on tender leaves, fruit, or panicles, finally producing downy white sporangiophores during late infection, which leads to major pre- and postharvest decay of the fruit, resulting in considerable economic loss (Jing et al. 2013). Efficient control of litchi downy blight is mainly based on the application of agrochemicals, including traditional fungicides, such as mancozeb, cymoxanil, and metalaxyl, as well as the recently registered Qol fungicide azoxystrobin (Wang et al. 2009b; Zhou et al. 2015).

${ }^{\dagger}$ Corresponding author: Q. Chen; qhchen@ hainanu.edu.cn

Hengyuan Guo and Jiandong Bao contributed equally to this work.

The author(s) declare no conflict of interest.

Accepted for publication 26 January 2021. copyright law, including all related and neighboring rights, to the extent allowed by law. 2021

\section{Funding}

This work was supported by grants from the National Natural Science Foundation of China (31772141), and the Scientific Research Foundation of Hainan University [KYQD(ZR)-20080], and the Innovation Team of Plant Protection, Fujian Academy of Agricultural Sciences (STIT2017-1-8).

\section{Keywords}

genomics, litchi downy blight, pathogenicity, Peronophythora litchii 
Table 1. Genome features of Peronophythora litchii

\begin{tabular}{|c|c|}
\hline Feature $^{a}$ & Strain ZL2018 \\
\hline ONT long reads & 10.89 Gb (approximately 170x) \\
\hline $\mathrm{N}_{50}$ of ONT Reads & $21.37 \mathrm{~kb}$ \\
\hline Mean ONT Read length & $15.57 \mathrm{~kb}$ \\
\hline Maximum ONT read length & $166 \mathrm{~kb}$ \\
\hline Illumina short reads ${ }^{b}$ & 5.10 Gb (approximately $73 x$ ) \\
\hline Estimated genome size & $57.08 \mathrm{Mb}$ \\
\hline Estimated genome repeats & $34.93 \%$ \\
\hline Estimated genome heterozygosity & $0.485 \%$ \\
\hline Assembly size (Mb) & $64.15 \mathrm{Mb}$ \\
\hline Contig number & 81 \\
\hline Contig $\mathrm{N}_{50}(\mathrm{Mb})$ & $1.43 \mathrm{Mb}$ \\
\hline Contig $L_{50}$ & 14 \\
\hline Average contig length & $0.79 \mathrm{Mb}$ \\
\hline Maximum contig length & $4.74 \mathrm{Mb}$ \\
\hline GC content & $49.72 \%$ \\
\hline Repeat sequence & $34.67 \%$ \\
\hline Illumina reads mapping rate & $92.47 \%$ \\
\hline Protein-coding genes ${ }^{c}$ & 14,857 \\
\hline Candidate RxLR effectors & 306 \\
\hline Genes annotated by Pfam & 14,385 \\
\hline Genes annotated by GO & 4,270 \\
\hline Genes annotated by KEGG & 6,629 \\
\hline Genes annotated by KOG & 10,307 \\
\hline Genes annotated by CAZy & 334 \\
\hline
\end{tabular}

${ }^{\mathrm{a}}$ ONT = Oxford Nanopore Technologies.

${ }^{\mathrm{b}}$ Genome heterozygosity, repeat content, and size were estimated by kmer-based method GenomeScope V2.0 using Illumina short reads.

${ }^{\mathrm{c}}$ Protein Family (Pfam) was annotated by InterProScan, while gene ontology (GO), Kyoto Encyclopedia of Genes and Genomes (KEGG), EuKaryotic Orthologous Groups (KOG), and carbohydrateactive enzyme (CAZy) were annotated by eggNOG-mapper.

The first genome assembly of $P$. litchi SHS3 was recently performed using Illumina short-read sequencing technology (Ye et al. 2016). However, the reported genome had an assembly size of only $38 \mathrm{Mb}$, which was much smaller than the estimated genome size (58 $\mathrm{Mb}$ ), and had fragmented into 2,543 scaffolds, potentially losing key genetic information such as interspersed repeats, copy number variation, and gene clusters.

To address this problem and generate a high-quality genome assembly resource for P. litchi, strain ZL2018 was isolated from Fujian Province in China. High-quality genomic DNA was extracted from mycelia and was sequenced using both third-generation sequencing (Oxford Nanopore Technologies [ONT]) and next-generation sequencing (NGS) (Illumina) technology at Biomarker Technologies Corp. (Beijing). For ONT long-read sequencing, a 20-kb genomic DNA library was constructed and sequenced using the ONT PromethION platform. We obtained $10.89 \mathrm{~Gb}$ (approximately $170 \times$ coverage) of long reads, with an average length of $15.57 \mathrm{~kb}, \mathrm{~N}_{50}$ of $21.37 \mathrm{~kb}$, and maximum length of $166 \mathrm{~kb}$ (Table 1). For Illumina short-read sequencing, a 350-bp genomic DNA library was prepared and 150-bp paired-end reads (PE150) were sequenced using the Illumina HiSeq 3000 platform, generating $5.10 \mathrm{~Gb}$ of short reads (approximatetely $73 \times$ coverage) (Table 1 ).

We performed k-mer-based genome size estimation of the Illumina short reads, using GenomeScope ver. 2.0 and KMC ver. 3.1.1. We identified the highest peak in the k-mer distribution histogram $(k=25)$ at the average k-mer depth of 63 , a smaller peak at twice the depth of the main peak (123, representing a repeat sequence), and another peak at half of the main peak depth (31, representing a heterozygous sequence). The estimated genome size was $57.14 \mathrm{Mb}$, which is consistent with the genome size of $58 \mathrm{Mb}$ estimated in a previous study (Ye et al. 2016). The estimated proportion of repeat sequences was $34.93 \%$ and the estimated heterozygosity was extremely low at $0.485 \%$ (Table 1 ).

High-continuity de novo genome assembly of strain ZL2018 was assembled using NextDenovo ver. 2.3.1 with ONT long reads, followed by correction using NextPolish ver. 1.3.0 with both ONT long reads and Illumina short reads. The final polished assembly was 64.15 $\mathrm{Mb}$ in size and consisted of 81 contigs with an $\mathrm{N}_{50}$ of $1.43 \mathrm{Mb}$, average length of $0.79 \mathrm{Mb}$, 


\section{BUSCO Assessment Results}

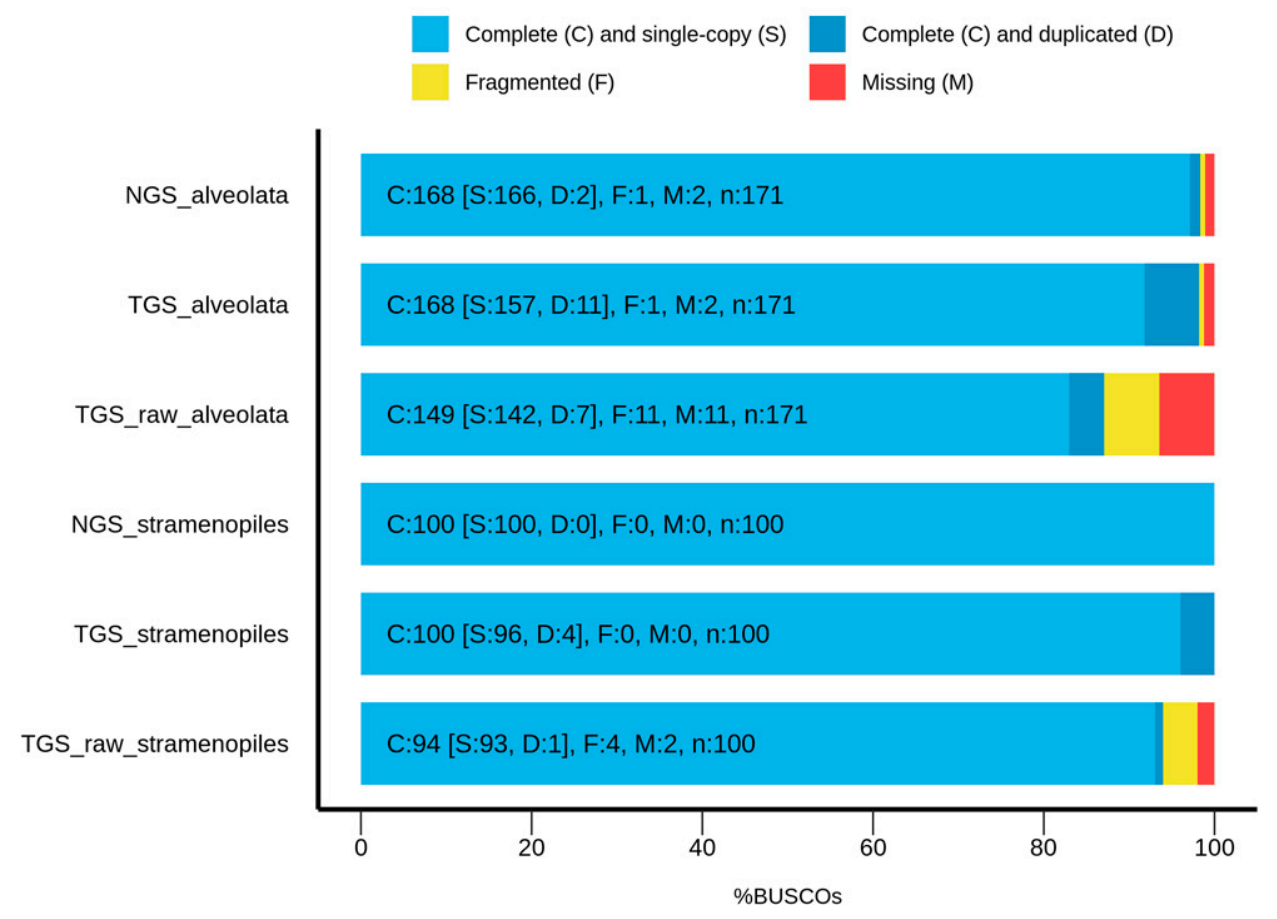

Fig. 1. Genome completeness evaluated by BUSCO v4.1.4 with Alveolata and Stramenopiles datasets. NGS means previous reported genome assembly of strain SHS3 generated by next generation sequencing, while TGS means our genome assembly of strain ZL2018 generated by third generation sequencing. TGS_raw indicates the unpolished draft genome assembly and TGS_polished indicates final error-corrected genome assembly.

and maximum length of $3.93 \mathrm{Mb}$ (Table 1). The genome assembly size was consistent with the estimated genome size $(57.14 \mathrm{Mb})$. Compared with the previously reported genome assembly, our genome was about $70 \%$ larger, with approximately $97 \%$ fewer contigs ( 81 vs. 2,543 ) and a 43-fold higher contig $\mathrm{N}_{50}(1.429 \mathrm{Mb}$ vs. $33 \mathrm{~kb})$.

Genome completeness was assessed using Benchmarking Universal Single-Copy Orthologs (BUSCOs) in Alveolata $(n=171)$ and Stramenopiles $(n=100)$ levels and BUSCO ver. 4.14 software. The final polished assembly contained $168(98.25 \%)$ complete and one fragmented orthologs in the Alveolata dataset and 100 (100\%) complete orthologs in the Stramenopiles dataset. It has been greatly improved from the unpolished draft genome assembly (87.13\% in the Alveolata dataset and $94 \%$ in the Stramenopiles dataset) (Fig. 1). In comparison with the previous reported genome assembly generated by NGS, the final polished assembly had the same number of complete orthologs in both datasets but contained more duplicated orthologs, which revealed higher genome completeness (Fig. 1). The completeness of the genome assembly was also evaluated by mapping Illumina short reads, which showed that a total of $92.47 \%$ reads were properly mapped (Table 1 ). These results represent solid evidence supporting the high continuity and completeness of our assembly.

Repeats in the genome assembly were de novo-annotated and masked using RepeatModeler ver. 2.01 and RepeatMasker ver. 4.1.1, respectively. Notably, $34.67 \%$ of the genomic sequences were repetitive (Table 1). Soft repeat-masked genome assembly was applied for protein-coding gene (PCG) prediction, using BRAKER2 software, which integrated ab initio and homology-based gene prediction, using proteins from Phytophthora sojae as a training dataset. A total of 14,857 PCGs were identified and 306 candidate RxLR effectors were identified using an open reading frame (ORF)-based method (Ye et al. 2016) (ORFs contain a signal peptide, RxLR motif in a 20- to 120-amino acid region and without transmembrane helices). Functional annotation analysis conducted using InterProScan ver. 5.47 .82 and eggNOG-mapper ver. 2 resulted in a total of $14,447(97.24 \%)$ genes annotated using the Pfam 14,385; 96.87\%), gene ontology $(4,270 ; 28.74 \%)$, Kyoto Encyclopedia of Genes and Genomes (6,629; 44.62\%), EuKaryotic Orthologous Groups (10,307; 69.37\%), and carbohydrate-active enzyme (334; $2.25 \%$ ) databases (Table 1). 
The whole-genome sequence data and raw sequence reads reported in this paper have been deposited in the Genome Warehouse (GWHAOTU00000000) and Genome Sequence Archive (CRA003810), respectively, in the National Genomics Data Center, Beijing Institute of Genomics (China National Center for Bioinformation), Chinese Academy of Sciences (BioProject PRJCA003784).

\section{Author-Recommended Internet Resources}

BRAKER2 software: https://github.com/Gaius-Augustus/BRAKER

BUSCO ver. 4.14 software: https://busco.ezlab.org

eggNOG-mapper ver. 2: http://eggnog-mapper.embl.de

Genome Warehouse: http://bigd.big.ac.cn/gwh

Genome Sequence Archive: http://bigd.big.ac.cn/gsa

GenomeScope ver. 2.0: https://github.com/tbenavi1/genomescope2.0

InterProScan ver. 5.47.82: https://github.com/ebi-pf-team/interproscan

$\mathrm{KMC}$ ver. 3.1.1: https://github.com/refresh-bio/KMC

NextDenovo ver. 2.3.1 and NextPolish ver. 1.3.0: https://github.com/Nextomics

RepeatMasker ver. 4.1.1: http://www.repeatmasker.org

RepeatModeler ver. 2.01: http://www.repeatmasker.org/RepeatModeler

\section{Literature Cited}

Ann, P. J., and Ko, W. H. 1984. Blossom blight of litchi in Taiwan caused by Peronophythora litchii. Plant Dis. 68:826.

Chi, P. K., Pang, X. P., and Liu, R. 1984. On downy blight of Litchi chinensis Sonn. The pathogen and its infection process. Acta Phytopathol. Sin. 2:113-119.

Huang, H., Wang, C. P., and Xu, D. Y. 1983. On Peronophythora litchii. Acta Mycologica Sin. 2:201-206.

Jing, G. X., Huang, H., Yang, B., Li, J. R., Zheng, X. L., and Jiang, Y. M. 2013. Effect of pyrogallol on the physiology and biochemistry of litchi fruit during storage. Chem. Cent. J. 7:19-29.

Kao, C. W., and Leu, L. S. 1980. Sporangium germination of Peronophythora litchii, the causal of litchi downy blight. Mycologia 72:737-748.

Vien, N., Benyon, F. H. L., Trung, H., Summerell, B. A., Van, N., and Burgess, L. W. 2001. First record of Peronophythora litchii on litchi fruit in Vietnam. Australas. Plant Pathol. 30:287-288.
Wang, H. C., Sun, H. Y., Stammler, G., Ma, J. X., and Zhou, M. G. 2009a. Baseline and differential sensitivity of Peronophythora Litchii (lychee downy blight) to three carboxylic acid amide fungicides. Plant Pathol. 58:571-576.

Wang, H. C., Sun, H. Y., Ma, J. X., Stammler, G., and Zhou, M. G. 2009b. Fungicide effectiveness during the various developmental stages of Peronophythora litchii in vitro. J. Phytopathol. 157:407-412.

Ye, W., Wang, Y., Shen, D., Li, D., Pu, T., Jiang, Z., Zhang, Z., Zheng, X., Tyler, B., and Wang, Y. 2016. Sequencing of the litchi downy blight pathogen reveals it is a Phytophthora species with downy mildew-like characteristics. Mol. Plant-Microbe Interact 29:573-583.

Zhou, Y., Chen, L., Hu, J., Duan, H., Lin, D., Liu, P., Meng, Q., Li, B., Si, N., Liu, C., and Liu, X. 2015. Resistance mechanisms and molecular docking studies of four novel Qol fungicides in Peronophythora litchi. Sci. Rep. 5:17466. 